\title{
Characteristics of the raindrop distributions in RICO shallow cumulus
}

\author{
O. Geoffroy ${ }^{1}$, A. P. Siebesma ${ }^{2}$, and F. Burnet ${ }^{1}$ \\ ${ }^{1}$ CNRM-GAME, Toulouse, France \\ ${ }^{2}$ KNMI, De Bilt, Holland \\ Correspondence to: O. Geoffroy (olivier.geoffroy@meteo.fr)
}

Received: 22 November 2013 - Published in Atmos. Chem. Phys. Discuss.: 9 January 2014

Revised: 13 August 2014 - Accepted: 22 August 2014 - Published: 15 October 2014

\begin{abstract}
The physical properties of rain spectra are generally modeled using an analytical distribution. It is common for the gamma distribution and, to a lesser extent, the lognormal distribution to be used. The majority of studies in the literature focusing on the characterization of raindrop distribution are based on deep convective cloud observations, mostly at ground level. This study focuses on shallow-cumulus rain distributions throughout the depth of the cloud layer and subcloud layer using airborne in situ measurements made with both the Particle Measuring Systems (PMS) Optical Array Probe 260X (OAP-260-X) and the PMS two-Dimensional Precipitation (2DP) instruments during the Rain in Cumulus over the Ocean (RICO) field experiment. Sampled spectra analyzed on the scale of large-eddy simulation resolution $(100 \mathrm{~m})$ are found to be relatively broad, with values of the shape parameter $-v$ for the gamma law and $\sigma_{\mathrm{g}}$ for the lognormal law - on the order of 1-3 and 1.5-2, respectively. The dependence of the shape parameters on the main rain variables (number concentration, water content, mean volume diameter, sedimentation fluxes and radar reflectivity) is examined, and a parameterization of the shape parameters $v$ and $\sigma_{\mathrm{g}}$ as a function of a power law of the rainwater content and raindrop number concentration is proposed.
\end{abstract}

\section{Introduction}

Raindrops play a role in the lower troposphere water and energy budgets by carrying water and latent energy from the cloud layer to the subcloud layer and to the surface. Assuming spherical raindrops, the physical properties of the raindrop field can be represented by the raindrop size (or mass) distribution on local scales, i.e., on scales on the order of a few dozen meters. The evolution of the raindrop size distribution depends on the interaction of various processes. In warm clouds, droplet growth is driven by condensation until its collection efficiency with respect to other cloud droplets starts to be significant, i.e., for diameters on the order of $40 \mu \mathrm{m}$. For a drop that reaches such a limit, called a precipitation embryo, the drop growth rate is exclusively the result of the collision-coalescence process and is roughly a function of the diameter to the power of six. The transition between these two regimes is highly nonlinear. The growth of the drops is limited, on the one hand, by the amount of cloud water available. On the other hand, large-drop formation is limited by two microphysical processes: collisioninduced breakup and spontaneous breakup. The latter occurs for diameters of a value on the order of about $10 \mathrm{~mm}$ (Pruppacher and Pitter, 1971). Both breakup processes contribute to a broadening of the raindrop distribution. The effect of collision-coalescence-breakup processes leads to an equilibrium distribution in around $1 \mathrm{~h}$ (Hu and Srivastava, 1995), which corresponds to about twice the lifetime of a shallowcumulus cloud cell. In unsaturated regions, the raindrop spectra evolve as the result of evaporation. In addition to these processes, the sedimentation process redistributes the raindrop sizes in the vertical: because large drops fall faster, the raindrop distribution tends to favor larger drops at lower levels (Milbrandt and Yau, 2005; Seifert, 2008). Thus, assuming a continuous and steady production of rain at cloud top, the rain distribution at a given level is in steady state only if the lifetime of the precipitating event is long enough to counteract the sedimentation size sorting effect. Ultimately, the local raindrop distribution is the result of a coupling between 
advection, turbulent transport and microphysical processes: collision,coalescence, breakup and sedimentation in cloud in a first stage; evaporation, sedimentation and, to a lesser extent, collision, coalescence and breakup out of the cloud in a second stage.

According to some hypotheses, each microphysical main rain variable and process can be directly expressed or parameterized as a function of the integral variables of the rain distribution, mostly moments. The moment of the order $p M_{p}$, is defined as follows:

$M_{p}=\int D^{p} n(D) \mathrm{d} D$

where $D$ is the particle diameter and $n(D)$ is the volume number density of raindrops with a diameter between $D$ and $\mathrm{D}+\mathrm{dD}$. The raindrop number concentration $N_{\mathrm{r}}$ is the zeroth moment of the distribution. The rainwater content $q_{\mathrm{r}}$ is proportional to the third moment of the distribution. Both are prognostic variables in two-moment bulk schemes. In radiative transfer calculation, the extinction is proportional to the second moment. The radar reflectivity, which is a useful quantity for remote-sensing measurements, is proportional to the radar reflectivity factor. Assuming Rayleigh scattering, the radar reflectivity factor is the sixth moment of the distribution (Smith et al., 1975). The collection of cloud droplets by raindrops (accretion) is usually parameterized as the product of cloud and rainwater contents (Kessler, 1969). The raindrop terminal velocity is roughly proportional to the diameter to the power of 0.8 . Thus the sedimentation fluxes of the rain concentration and the rainwater content vary as a linear function of the moments 0.8 and 3.8, respectively. Hence, they are roughly dependent on $M_{1}$ and $M_{4}$. The evaporation rate is the sum of two linear functions depending roughly on the moments of the order 0.8 and 1.8 .

Since only a limited number of rainfall integral variables are generally known (e.g., $M_{0}$ and $M_{3}$ in two-moment bulk schemes, $\mathrm{M}_{6}$ in remote-sensing measurement), a hypothesis on the shape of the distribution is necessary in order to derive the other microphysical properties. Raindrop distributions are generally represented by the exponential law (Marshall and Palmer, 1949), hereafter referred to as MP distribution, or by a gamma distribution function (Ulbricht, 1983). The latter is expressed as

$n(D)=N \frac{1}{\Gamma(v)} \lambda^{v} D^{\nu-1} \exp (-\lambda D)$.

It has three independent parameters: the number concentration $N$, the slope parameter $\lambda$ and the shape parameter $\nu$. The gamma law is a general case of the exponential function $(v=1)$. Note that the most common expression used for the shape parameter is $\mu=v-1$ rather than $v$. The latter is used in this study because it is defined on ] $0,+\infty$ [, which permits plots on the logarithmic scale. The slope parameter $\lambda$ is related to the mean volume diameter $D_{\mathrm{v}}$ and $v$ in the following way:

$\lambda=\frac{1}{D_{\mathrm{v}}}(v(v+1)(v+2))^{1 / 3}$.

In some studies the lognormal distribution is assessed (Feingold Levin, 1986):

$n(D)=N \frac{1}{\sqrt{2 \pi} D \ln \sigma_{\mathrm{g}}} \exp \left(-\frac{1}{2}\left(\frac{\ln \left(D / D_{\mathrm{g}}\right)}{\ln \sigma_{\mathrm{g}}}\right)^{2}\right)$,

where $\sigma_{\mathrm{g}}$ is the geometric standard deviation and $D_{\mathrm{g}}$ is the mean geometric diameter.

The benefit of using these distributions is that each moment of the distribution can be analytically calculated as a function of the three parameters. In a two-moment bulk scheme, two parameters are imposed by the prognostic variables and one remains to be fixed: $v$ for the gamma and $\sigma_{\mathrm{g}}$ for the lognormal distribution. Figure 1 shows the moments of the order 1,2, 4 and 6 as a function of the shape parameters for fixed concentration $\left(M_{0}\right)$ and water content $\left(M_{3}\right)$. When vincreases, the distribution is narrower: $M_{p}$ increases with $v$ for $\mathrm{p}<3$, decreases for $p>3$ and vice versa for the lognormal law. For $v>10$ or $\sigma_{\mathrm{g}}<1.1$, each moment does not vary significantly because the distribution tends to the monodispersed distribution. Note that, in this study, narrow (broad) refers to spectra with a high (low) value of $v$ or a low (high) value of $\sigma_{\mathrm{g}}$ and not to high standard deviation values, which also depend on the mean volume diameter.

Since the work of Marshall and Palmer (1949) and Best (1950), a large number of studies have been dedicated to the retrieval of the value of these parameters characteristic of deep convective events. Most of these studies suggest that rain spectra are narrower than the MP distribution $(v=1)$, with $v$ values roughly in the range of 5-10 (Nzeukou et al., 2003; Uijlenhoet et al., 2003) or more (Tokay and Short, 1996) or $\sigma_{\mathrm{g}}$ values on the order of 1.4 (Feingold and Levin, 1986). These studies are based on $1 \mathrm{~m}$ surface measurements with the RD-69 disdrometer (Joss and Waldvogel, 1967). Ulbrich and Atlas (1997) airborne 2-D precipitation probe measurements at $6 \mathrm{~s}$ resolution suggest broader spectra, with a mean value of $5(\mu=v-1=6)$, than the Tokay and Short (1996) mean value of 11 for the same field experiment. By analyzing $1 \mathrm{~m}$ resolution spectra derived from video disdrometer measurements at the surface, Brandes et al. (2003) also found broad spectra, with most values falling between the MP value and $v=5$. Van Zanten et al. (2005) found narrow drizzle spectra in stratocumulus despite the coarse resolution of $2 \mathrm{~m}$, with $\sigma_{\mathrm{g}}$ values on the order of 1.5 1.8 .

Studies diverge not only with regard to the magnitude of the shape parameter values but also concerning their relationship with other variables. Experimental studies show a positive correlation between $v$ and the precipitation flux (Tokay and Short, 1996; Cerro et al., 1997; Nzeukou et al., 2004) and numerical studies point to the narrowing of the spectra 
with increasing mean volume diameter induced by size sorting (Milbrandt and Yau, 2005; Seifert, 2008). On the other hand, PRECIP98 measurements show a negative correlation between $v$ and the precipitation flux and between $v$ and the mean volume diameter (Zhang et al., 2001). All these studies focused on deep precipitating, stratiform or boundary layer clouds. The lack of convergence between studies suggests a different type of rain spectra according not only to the type of cloud but also to the location in the cloud system, the methodology employed, the temporal and horizontal resolutions, the instruments used, and instrumental biases. Until now, no study has assessed the shape parameter in shallowcumulus convection.

In this study, the representation of the rain spectra in shallow cumulus is examined via the values of the shape parameters $\sigma_{\mathrm{g}}$ and $v$. The following section describes the data set and gives an insight into the vertical profiles of the measured precipitation fields; the shape parameter analysis results are reported in Sect. 3 .

\section{Data set and vertical structure of the precipitation field}

The observations used in this study are derived from in situ shallow precipitating cumulus cloud measurements collected during the RICO field experiment (Rauber et al., 2007; Snodgrass, 2008; Nujiens et al., 2009). Two instruments are combined to retrieve the complete raindrop size distribution. The Particle Measuring Systems (PMS) Optical Array Probe 260X (OAP-260-X) provides droplet and drizzle size from $5 \mu \mathrm{m}$ to $635 \mu \mathrm{m}$ over 63 bins of $10 \mu \mathrm{m}$ bin width. The PMS two-Dimensional Precipitation (2DP) measures the diameter of larger drops over 32 or 64 bins of $200 \mu \mathrm{m}$ bin width between $100 \mu \mathrm{m}$ and an upper limit depending on the method used by the NCAR to construct the particle spectra from the PMS 2DP images.

The entire-in method takes into account only particles that fully cross the sampling section and assumes that the diameter is the drop thickness along the diode array (Heymsfield et al., 1978). The sampling volume decreases with drop diameter because the upper limit of the measured diameter is restricted by the thickness of the diodes, which is on the order of $6 \mathrm{~mm}$. The center-in method also takes into account partially sampled drops by accounting for all particles for which the center is within the sampling section. The diameter of the raindrop is assumed to be the maximum value of the width of the raindrop along the flight path and its thickness. This method increases the 2DP sampling volume and allows larger drops, up to $12700 \mu \mathrm{m}$, to be taken into account.

Large raindrop diameters are especially subject to being biased due to their nonspherical shape (Pruppacher and Beard, 1970; Chandrasekar et al., 1988), to the very low number of such particles and to spurious counts (Heymsfield and Baumgardner 1985; Backer et al., 2009). Thus, the
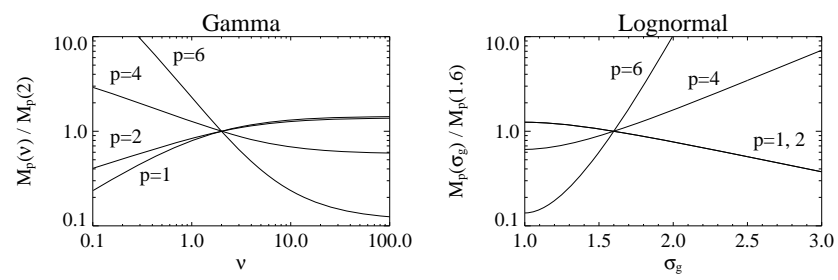

Figure 1. Relationship between the moments of the order $p=$ $1,2,4$ and 6 , and the shape parameter for the gamma function (left) and the lognormal function (right). Each moment is normalized by the value corresponding to $v=2$ for the gamma function and $\sigma_{g}=1.6$ for the lognormal function.

center-in spectra are used in this study, and sensitivity tests are performed according to the method used in Sect. 3.

For data processed at $1 \mathrm{~Hz}$, that is, a resolution of about $100 \mathrm{~m}$ along the flight track, the sampled volume is on the order of 1-4 L and 100-200 L for the PMS OAP-260X and the PMS 2DP, respectively. This is low compared to the typical value of raindrop number concentration, which is about $0.1-100 \mathrm{~L}^{-1}$. To increase the representativeness of the sample, one can cumulate counts over a larger distance. However, because of the heterogeneity of the raindrops' spatial distribution, the shape of the spectra is sensitive to the resolution. An increase in the sample length broadens the spectra.

The lower limit of the raindrop spectra $D_{0}$, which corresponds to the separation diameter between cloud droplet and drizzle, is assumed to be $75 \mu \mathrm{m}$. Sensitivity tests have shown that the results presented here are not sensitive to this threshold, at least over the range of $50-100 \mu \mathrm{m}$. Spurious counts, which affect both low and high diameters (Backer et al., 2009), are removed in 2DP and OAP-260-X measurements. Similarly to Yuter and Houze (1997), all nonconsecutive bins above $1500 \mu \mathrm{m}$ are set to 0 , and the isolated positive bins in OAP-260-X are excluded. Because the moments of the distribution are sensitive to the extremities of the distribution, further sensitivity tests are performed in Sect. 3. Finally, the first bin of the PMS 2DP is removed to avoid overlap with the OAP-260-X measurements.

Of the 19 RICO flights analyzed in this study, 13 are characterized by significant rainy events (RF01, RF03, RF04, RF05, RF07, RF08, RF10, RF11, RF13, RF14, RF15, RF16, RF19) and 6 are rejected due to the insignificant number of rain samples (RF02, RF06, RF09, RF12, RF17, RF18). Rain spectra are defined here as samples with rainwater content $q_{\mathrm{r}}>0.010 \mathrm{~g} \mathrm{~m}^{-3}$. The total number of precipitating samples at $1 \mathrm{~Hz}$ resolution is about 21000 . During RICO, the NCAR C-130 aircraft flew through the cloud field at different altitudes between about $100 \mathrm{~m}$ and $3 \mathrm{~km}$. To distinguish between in-cloud and clear-sky samples, we used data from the fast forward-scattering spectrometer probe (FFSSP) instrument (Brenguier et al., 1998) that provides a droplet size distribution from $\sim 2$ to $50 \mu \mathrm{m}$ in diameter for the flights RF07, 08 and 11 . 

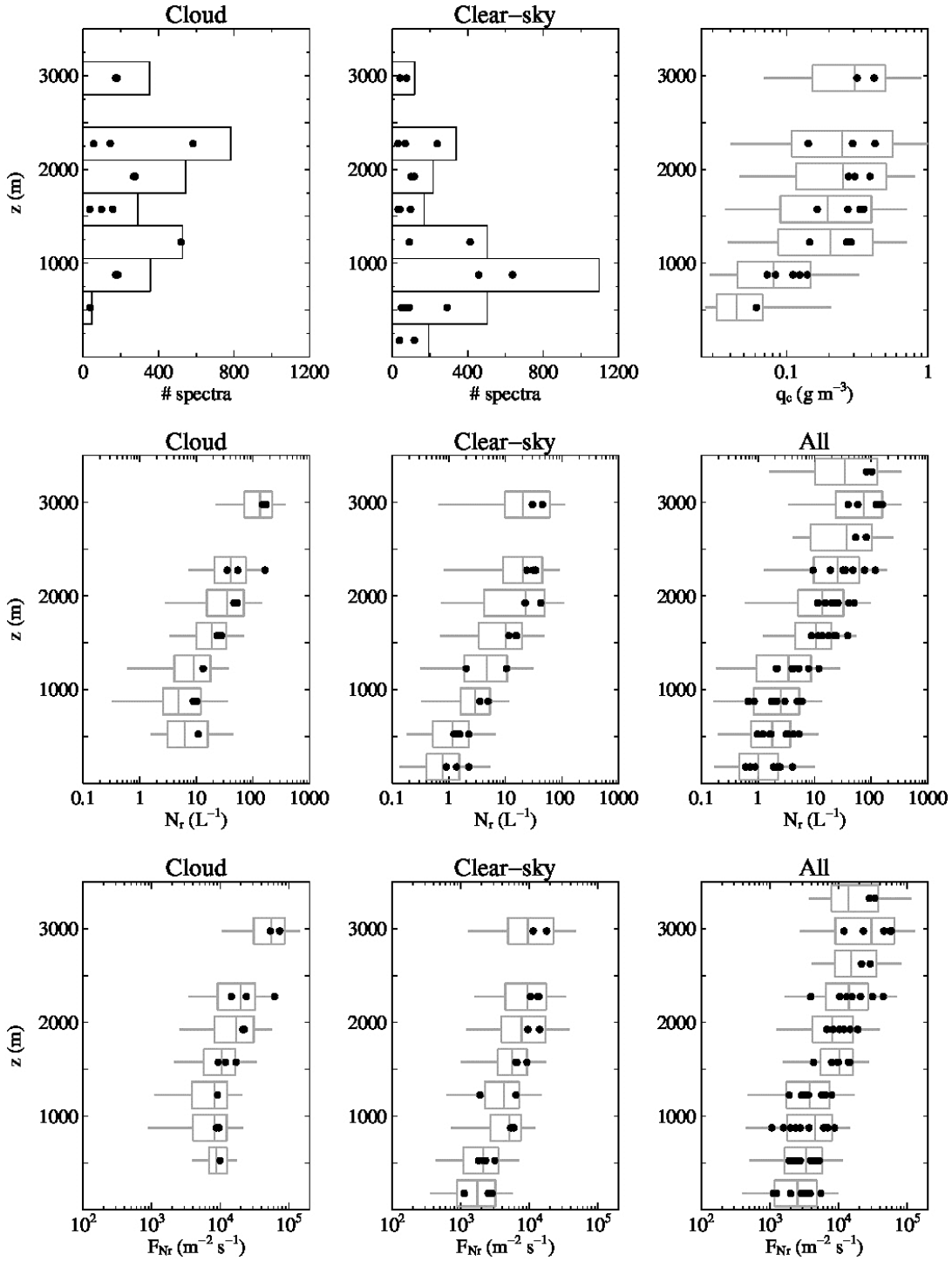

Figure 2. Total number of rain-sampled spectra in the cloud region (top row, left) and in clear sky (top row, center). Vertical profile of statistical distribution of cloud water content $q_{\mathrm{c}}$ (top row, right) sampled at $1 \mathrm{~Hz}$ for flights with FFSSP measurements available, and vertical profile of statistical distribution of the rain variables sampled at $1 \mathrm{~Hz}$ for the rain concentration $N_{\mathrm{r}}$, the rain concentration flux $F_{\mathrm{Nr}}$, the rainwater content $q_{\mathrm{r}}$, the precipitation flux $F_{\mathrm{qr}}$ and the rain mean volume diameter $D_{\mathrm{v}}$, in the cloud region (left) and in the clear-sky region (middle), for flights with FFSSP available, and in all regions (right). The box plots denote the 5th, 25th, 50th, 75th and 95th percentiles of the variable distribution in every $300 \mathrm{~m}$ layer. Filled circles are mean values for each flight.

The vertical structure of the main rain variables is represented in Fig. 2. On the upper panel, the first two plots show the number of rain spectra sampled at each level of the lower troposphere, in cloudy air and in clear air. Because the aircraft was pointing towards the cloud cells, a large part of the rain spectra (almost $60 \%$ ) were sampled in clouds. The third plot shows the vertical profile of the cloud liquid-water content derived from the FFSSP data. The following panels show the profiles of the rain concentration $N_{\mathrm{r}}$, the rain number concentration flux $F_{\mathrm{Nr}}$, the rainwater content $q_{\mathrm{r}}$, the precipitation flux $F_{\mathrm{qr}}$ and the rain mean volume diameter $D_{\mathrm{v}}$. For each parameter, the first two profiles correspond to in-cloud and clear-sky samples (left and middle column, respectively) for the three flights with FFSSP data. The last profile (right-hand column) corresponds to the statistics of all samples of the 13 flights. Box plots with the 5th, 25th, 50th, 75th and 95th percentiles of the distribution are used to indicate the spread of the data. Symbols are mean values for each flight and are superimposed to illustrate the flight-to-flight variability.

Values are averaged over the rain fraction at the corresponding level. Hence, these profiles are not directly comparable to profiles averaged over the whole domain or over 

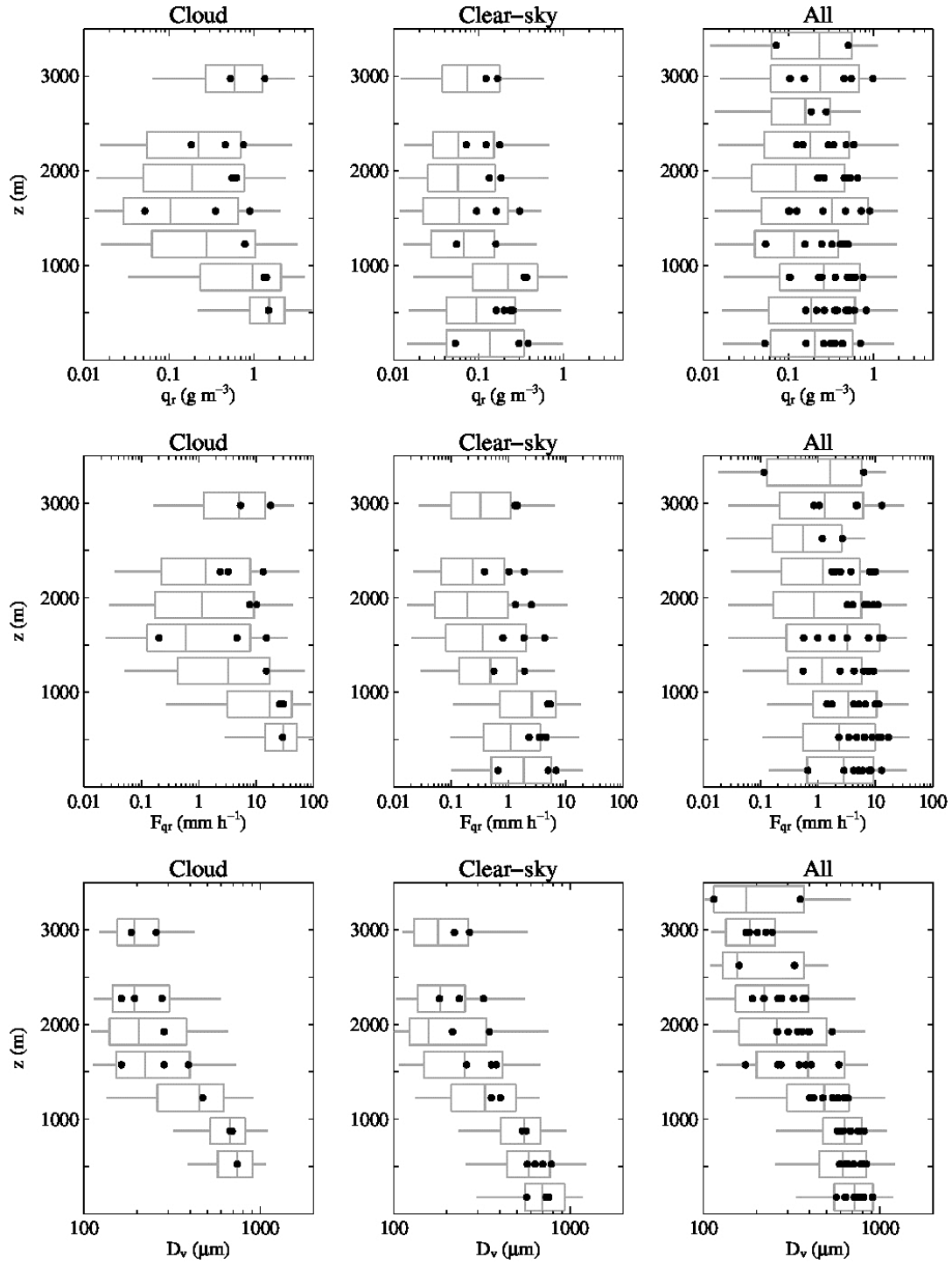

Figure 2. Continued.

the projected cloud fraction. Above the cloud base, some rain falls in clear sky. This feature may be due to the wind shear, to turbulent motions or to the fact that raindrops have a longer lifespan than cloud droplets. Such a pattern was reproduced by the Dutch atmospheric model DALES (Heus et al., 2010) large-eddy simulations (LES) simulations of shallow cumulus even without shear (not shown). However, in LES simulations, a large part of the rain mass falls in clear sky, which is not suggested here by the $q_{\mathrm{r}}$ and the $F_{\text {qr }}$ profiles. Finally, Fig. 2 reveals that all rain quantities are larger in clouds mainly due to evaporation that occurs in clear sky.

The profiles of $q_{\mathrm{r}}$ and $F_{\mathrm{qr}}$ do not show a particular trend with altitude. While evaporation leads to a decrease in their domain-average value, here values are averaged over the rain fraction, which decreases with height, compensating for the effects of evaporation. In contrast, the rain number concen- tration $N_{\mathrm{r}}$ (and the rain concentration flux) and the mean volume diameter $D_{\mathrm{v}}$ decrease and increase, respectively, with decreasing altitude. All processes (collection, evaporation, sedimentation) contribute to a decrease in the number concentration and in the rain concentration flux, which is consistent with these observations. The dispersion of the mean volume diameter is small, in particular in the subcloud layer, and it exhibits the same trend in cloudy air and in clear air, suggesting that its evolution is mainly driven by size sorting. The trends shown here are similar to those observed in drizzling stratocumulus clouds (Wood et al., 2005), except that, here, rain concentration and mean volume diameter also vary above the lifting condensation level, both in and outside the cloud.

In comparison to the results of van Zanten et al. (2010) (their Figure 8), the profiles show the same trends, with a 


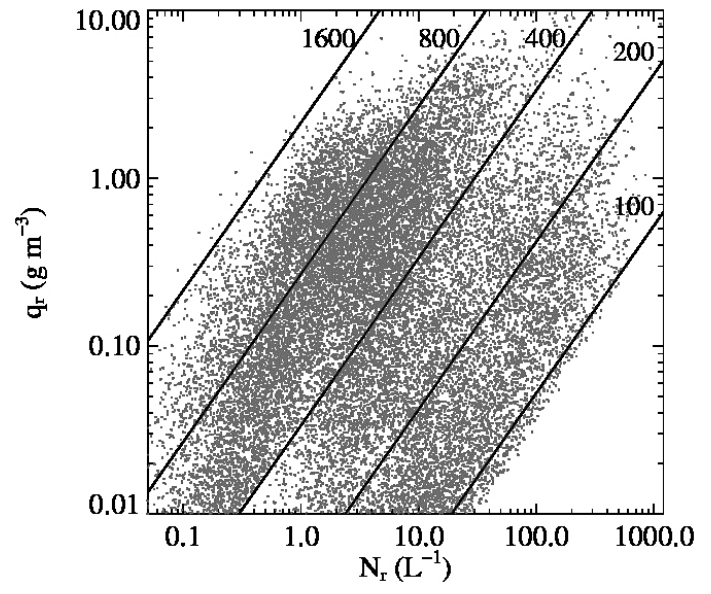

Figure 3. Scatterplot of the drop number concentration, $N_{\mathrm{r}}$, and the rainwater content, $q_{\mathrm{r}}$, for drop spectra sampled at $1 \mathrm{~Hz}$. Lines represent constant mean volume diameters for $D_{\mathrm{V}}=1600,800,400$, $200,100 \mu \mathrm{m}$.

pronounced increase in $N_{\mathrm{r}}$ with altitude while $q_{\mathrm{r}}$ remains more or less constant. However both profiles reveal higher values with median values of $N_{\mathrm{r}}$ and $q_{\mathrm{r}}$ ranging from 1 to $100 \mathrm{~L}^{-1}$ and from 0.1 to $0.3 \mathrm{gm}^{-3}$, respectively. These differences come from the cases selected here: nine precipitating cases have been added and three cases with a very low precipitation amount have been removed. It follows that the statistics are shifted to larger values as reflected by the flight average values. Note that the profiles presented here are closer to the simulations of the LES models reported in van Zanten et al. (2010) than the observed profiles they present. As shown by the box plots, the scatter of the rain variables is large, especially for the rainwater content that covers about two orders of magnitude. This scatter is due to the large heterogeneity of the rain field inside a given cloud system and to the differences in the microphysical and macrophysical properties of the sampled cloud systems. In boundary layer clouds, the strength of the precipitation production depends on both the cloud droplet concentration and liquid-water path or cloud depth (Geoffroy et al., 2008; Jiang et al., 2010, Burnet and Brenguier, 2010), both of which vary among the different flight cases. However, note that for the profiles of $N_{\mathrm{r}}$, $F_{\mathrm{Nr}}$ and $D_{\mathrm{v}}$, box plots and flight averages both follow the same pronounced vertical trend reflecting the consistency of the observations.

Some studies have examined the relationship between the slope parameter $\lambda$ and the shape parameter $v$ for remote-sensing retrieval of the rain distribution characteristics, mainly the precipitation flux, from radar measurements (Zhang et al., 2001; Chang et al., 2009). Atlas and Ulbricht (2006) suggest that there is no universal relationship that would describe all types of storm spectra accurately. The RICO measurements encompass a large range of rain microphysical properties and confirm this fact. Indeed, assuming that the gamma distribution gives an accurate representation of the rain spectra, $\lambda$ depends on $v$ and $D_{\mathrm{v}}$ (Eq. 3). Because the profile of $D_{\mathrm{v}}$ varies significantly with height, it follows that the $\lambda-v$ relationship depends necessarily on altitude.

This study is restricted to the estimation of the shape parameter of both lognormal and gamma laws, assuming that $N_{\mathrm{r}}$ and $q_{\mathrm{r}}$ are known, as is the case in a simulation using a two-moment bulk microphysics scheme. Figure 3 shows the space parameter of $N_{\mathrm{r}}$ and $q_{\mathrm{r}}$ for all RICO spectra at $1 \mathrm{~Hz}$ resolution. The reported values cover a large range of rain properties from drizzle, with about $50 \%$ of the drop concentration values greater than $5 \mathrm{~L}^{-1}$ and $10 \%$ greater than $50 \mathrm{~L}^{-1}$ to intense precipitating events with samples that have a high local rainwater content between 1 and $10 \mathrm{~g} \mathrm{~m}^{-3}$. The mean volume diameter ranges from $100 \mu \mathrm{m}$ to about $1 \mathrm{~mm}$ near the surface. Most of the measurements are performed inside clouds or close to clouds rather than in clear sky. As a result, the statistics are slightly biased toward initial stages of precipitation formation. Nevertheless, as attested by Fig. 3, the data set covers a large range of values; hence we assume in the following that it is representative of rain spectra in shallow cumulus.

\section{Shape parameters analysis results}

In this section, the ability of the lognormal and the gamma distributions to represent shallow-cumulus drop spectra is evaluated. The method used is the one detailed in Geoffroy et al. (2010) (hereafter G10) for cloud droplet spectra analysis. The raindrop spectra are assumed to be described by an analytical distribution. For each moment representative of a physical process $M_{1}, M_{2}, M_{4}$ and $M_{6}$, the shape parameter is calculated numerically by a minimization of the distance between the measured moment and the analytical moment. This method is similar to the commonly used method of moments (Waldvogel 1974; Ulbrich 1983) applied with $M_{0}, M_{3}$ and a third moment that is the one to be parameterized. It has the benefit of providing the exact value to use to represent a considered moment and avoiding negative values for $v$. Such negative values can be found by analytical calculation, for instance in Zhang et al. (2001). Some studies (Ulbrich and Atlas, 1998; van Zanten et al., 2005) consider truncated functions. However, the assumed distributions are not truncated when used in models or for remote-sensing parameter retrieval in order to avoid too many complex calculations. Furthermore, the use of complete distributions allows analytical integrations. For these reasons, this study is limited to complete functions. Moreover, such truncations do not significantly modify the results.

Figure 4 shows the shape parameters for each moment $\left(M_{1}, M_{2}, M_{4}\right.$ and $\left.M_{6}\right)$ estimation as a function of the considered moment. The number of samples in each moment class is represented in the lower row. The value of $v$ is represented on a log scale because of the strong dependence of moments 

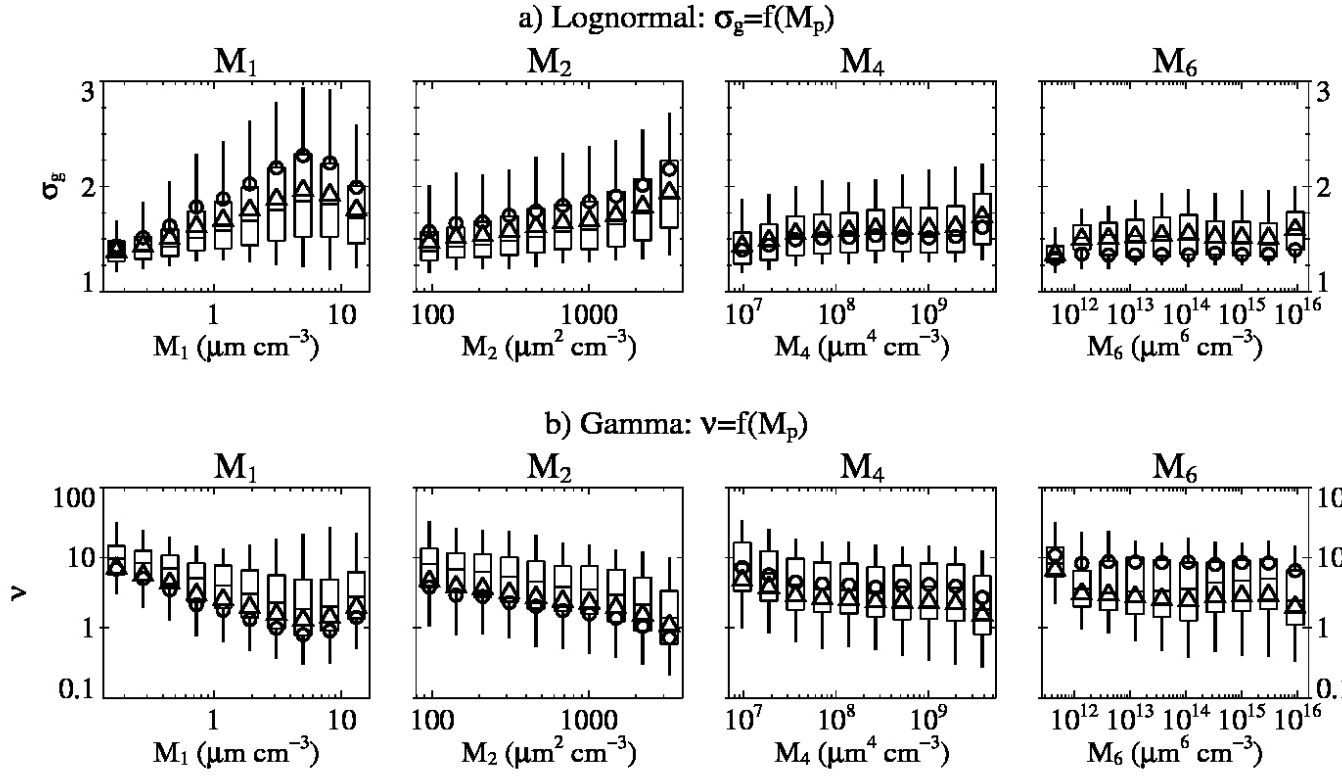

b) Gamma: $v=f\left(M_{p}\right)$
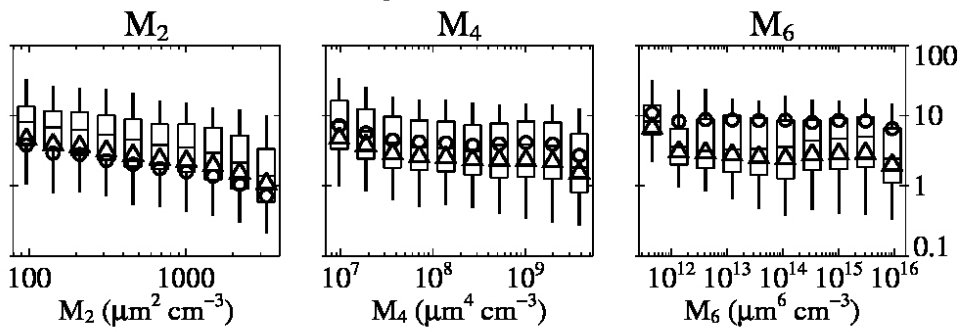

c) Spectra frequency distribution
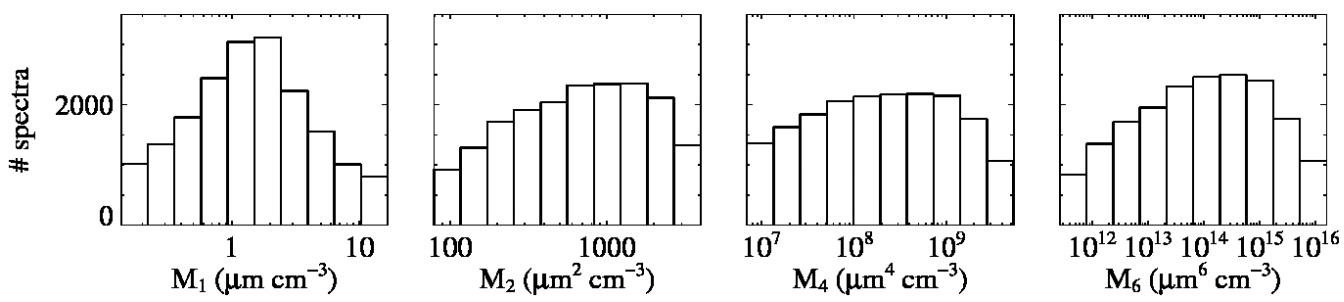

Figure 4. Statistical distribution of the shape parameter values as a function, from left to right, of the $M_{1}, M_{2}, M_{4}$ and $M_{6}$ moment values. The $x$ axis is divided into 10 classes on a log scale. The box plots denote the 5th, 25th, 50th, 75th and 95th percentiles of the shape parameter distribution in each class. The circles and triangles denote the tuning parameter value that minimizes the standard deviation of the absolute error and the geometric standard deviation of the log error in each class, respectively. The top and second rows are for the lognormal function and the gamma function, respectively. The third row shows the number of sampled spectra in each moment class.

on $\log (v)$ (Fig. 1). According to G10, the circles and triangles are the shape parameter values that minimize the arithmetic and the geometric standard deviation of the absolute and relative errors, respectively, in each moment class.

For each minimization, there is a strong scatter of the shape parameter. The values of $v$ range roughly from 1 to 10. As a general trend, we observe that, as the value of the considered moment increases spectra become narrower, as shown by the increase in $\sigma$ and the decrease in $v$ for both percentiles and the mean values. This trend is especially pronounced for the $M_{1}$ and the $M_{2}$ minimizations. The $M_{6}$ minimization gives narrower spectra on average, especially for the lognormal model, because of the highest dissymmetry of this function. However, high-order moments are sensitive to the presence of large drops. When spurious counts are not cleaned, broader spectra are obtained for the $M_{6}$ minimization. Despite the large scatter observed in the shape parameters and the dependence of the results on the chosen moment, data are merged in order to derive a trade-off value of the shape parameters and to determine a single law representative of all processes.

The trade-off values $v^{*}$ and $\sigma_{\mathrm{g}}^{*}$, of the gamma and the $\log$ normal law, respectively, are calculated by averaging the 80 optimum shape parameter values in each bin following G10 for the different resolutions of $1,0.5,0.2$ and $0.05 \mathrm{~Hz}$ (i.e., a distance on the order of $100,200,500$ and $2000 \mathrm{~m}$, respectively). The results are summarized in Table 1 .

A value of 3.2 for $\nu^{*}$ and 1.63 for $\sigma_{\mathrm{g}}^{*}$ is obtained from the cleaned spectra (noted E2). The broadness of the spectra increases when the resolution decreases, as expected, because of the high heterogeneity of the rain field. On the scale of the cloud cell, distributions are close to the MP distribution $(v=1)$. Table 1 also shows the arithmetic and geometrical means of each ensemble of shape parameter values. The geometric mean of the gamma law shape parameter $v$ and the arithmetic mean of the lognormal law shape parameter $\sigma_{\mathrm{g}}$ are close to the trade-off values $v^{*}$ and $\sigma_{\mathrm{g}}^{*}$, respectively. These results suggest that such methods of averaging (geometric 
Table 1. Values of $v^{*}$ and $\sigma_{\mathrm{g}}^{*}$, the arithmetic mean $\left(v_{\text {arith }}, \sigma_{\text {garith }}\right)$ and geometric mean $\left(v_{\text {geom }} \sigma_{\text {geom }}\right)$ of the ensemble of shape parameter values, as well as values of $v^{*}$ for spectra reconstructed using the center-in $(\mathrm{C})$ method, entire-in method (E) and spectra truncated above $1500 \mu \mathrm{m}(<1500)$, under $300 \mu \mathrm{m}(>300)$ and both (300-1500). All values are given for four resolutions: $1,0.5,0.2 \mathrm{and} 0.05 \mathrm{~Hz}$.

\begin{tabular}{lccccc}
\hline & & $1 \mathrm{~Hz}(\sim 100 \mathrm{~m})$ & $0.5 \mathrm{~Hz}(\sim 200 \mathrm{~m})$ & $0.2 \mathrm{~Hz}(\sim 500 \mathrm{~m})$ & $0.05 \mathrm{~Hz}(\sim 2000 \mathrm{~m})$ \\
\hline E2 & $\nu *$ & 3.2 & 2.7 & 2.2 & 1.6 \\
E2 & $<v>_{\text {geom }}$ & 3.5 & 3.0 & 2.5 & 1.8 \\
E2 & $<v>_{\text {arith }}$ & 6.7 & 5.5 & 4.4 & 3.2 \\
& & & & & 1.81 \\
E2 & $\sigma_{\mathrm{g}}^{*}$ & 1.63 & 1.67 & 1.72 & 1.76 \\
E2 & $<\sigma_{\mathrm{g}}>_{\text {geom }}$ & 1.59 & 1.63 & 1.68 & 1.79 \\
E2 & $<\sigma_{\mathrm{g}}>_{\text {arith }}$ & 1.62 & 1.66 & 1.71 & 1.0 \\
& & & & & 0.9 \\
E & $\nu *$ & 2.4 & 1.9 & 1.5 & 1.8 \\
C & $\nu *$ & 2.2 & 1.8 & 1.3 & 5.9 \\
E2 $<1500$ & $\nu *$ & 3.3 & 2.8 & 6.3 & 7.3 \\
E2 $>300$ & $\nu *$ & 8.0 & 7.6 & 8.1 & \\
E2 300-1500 & $\nu *$ & 9.0 & 8.6 & & \\
\hline
\end{tabular}

mean for the gamma and arithmetic mean for the lognormal) are adequate for estimating the shape parameter. Moreover, this result is consistent with the logarithmic and the linear dependency of the moments for the gamma and the lognormal laws, respectively. The arithmetic mean, generally used in studies to retrieve the characteristic $v$ value of the rain distributions, has significantly higher values.

To gain insight into the errors associated with the spurious count for both large and small drops and those associated with a lack of statistical representation, sensitivity tests to the tail of the rain spectra were performed. Without removing the spurious count, the entire-in method (E) and center-in method (C) give similar results, which suggest a low contribution of the drops larger than $6 \mathrm{~mm}$, with a $v^{*}$ value on the order of 2. This value should give a lower boundary for $v^{*}$. Truncations under $300 \mu \mathrm{m}$ in diameter (i.e., the use of only 2DP measurements), above $1.5 \mathrm{~mm}$ and both show that the shape parameter value is mostly sensitive to the presence of the smallest drops. Spectra obtained are narrower, with an extreme value of $v^{*}$ equal to 9 at $1 \mathrm{~Hz}$, which should give a comfortable estimation of its upper bound. Such truncated spectra are close to the Joss and Waldvogel (1967) disdrometer range. The $0.05 \mathrm{~Hz}$ value of $v^{*}$ is close to that derived from most previous studies.

The data for the shape parameter $v$ are shown on Fig. 5a-f as function of $N_{\mathrm{r}}, D_{\mathrm{v}}$ and $q_{\mathrm{r}}$, in order to examine the sensitivity of this shape parameter to variables predicted in twomoment bulk schemes. Only $M_{1}$ and $M_{4}$ moment values are presented here because they are the most important with respect to the parameterization purpose, especially for the sedimentation and the evaporation processes. The largest scatter in the sixth box plot of Fig. 5c, d corresponds to the transition between the OAP-200-X and the 2DP measurements, marked by an important decrease in the size resolution (from 10 to $200 \mu \mathrm{m})$. Measurements show a clear negative trend as a function of $q_{\mathrm{r}}$, as already depicted in Fig. 4. In contrast, no obvious trend is observed for $N_{\mathrm{r}}$ and $D_{\mathrm{v}}$ over the whole range. For both lowest and largest $D_{\mathrm{v}}$ values, $v$ is large (median values $>5$ ), corresponding to narrow size distributions. The broadest spectra correspond to large concentration values greater than about $4 \mathrm{~L}^{-1}$ and intermediate mean volume diameter values from about 200 to $400 \mu \mathrm{m}$, but with a large dispersion as reflected by the $25-75$ th percentile interval that could reach an order of magnitude.

At the early stage of the rain formation, samples are characterized by high concentration values, especially in the upper part of the cloud, as attested by Figure 2, low Dv values and narrow spectra. As drops grow by collision and coalescence and are mixed by turbulence (that is, in the highrainwater-content samples) the size spectra broaden and the mean volume diameter reaches intermediate values while the concentration decreases slightly but still remains relatively high. As a result, the flight average concentration values are larger than $10 \mathrm{~L}^{-1}$ above $1500 \mathrm{~m}$ as indicated by Fig. 2. Consequently, spectra with high concentration may be young narrow spectra characterized by low mean volume diameter or, by contrast, aged broad spectra with a large amount of rain. This explains the large scatter of $v$ for large concentration values. The vertical profiles of Fig. $5 \mathrm{~g}$, h show an increase in $v$ with decreasing altitude. This trend is more pronounced in the subcloud layer. It is consistent with experimental studies that show narrower distributions at the surface than in clouds (Tokay and Short, 1996; Ulbrich and Atlas, 1998) and with 1-D numerical studies focusing on the effect of size sorting (Milbrandt and Yau, 2005; Seifert, 2008).

The shape parameters retrieved here differ from those reported in previous studies that focused on deep convective events for similar spatial and horizontal resolutions. These discrepancies are likely due to differences in rain characteristics specific to the cloud regime. In shallow cumulus the 

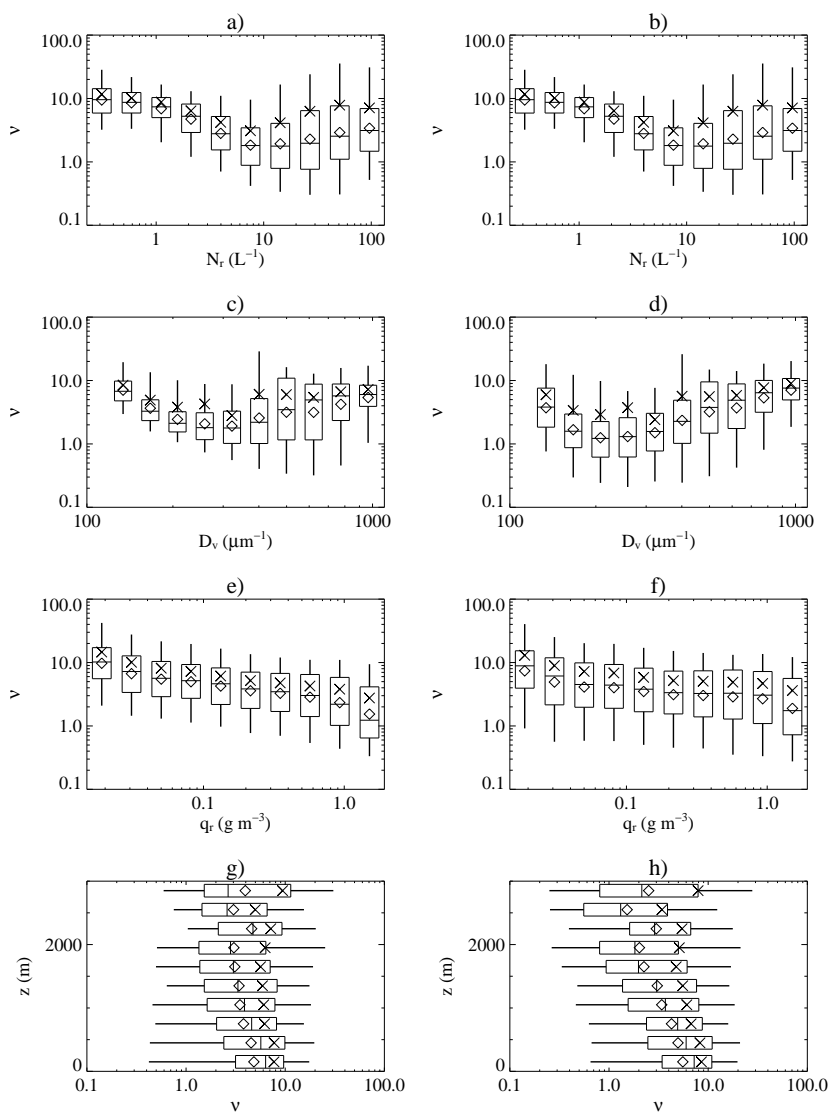

Figure 5. Statistical distribution of the shape parameter values as a function of rain number concentration $N_{\mathrm{r}}$ (upper line), rain mean volume diameter $D_{\mathrm{v}}$ (second line) and rainwater content (third line) and profile of the statistical distribution of the shape parameter values (lower line) for the $M_{1}$ minimization (left column) and the $M_{4}$ minimization (right column). The box plots denote the 5th, 25th, 50th, 75th and 95th percentiles of the shape parameter distribution in each class. The diamonds and crosses denote the arithmetic mean and the geometric mean in each class, respectively.

mean volume diameters are lower and the rain number concentrations are higher than in deeper clouds. They can also be partially attributed to instrumental limits, averaging procedures and the location of the samples. As in G10, Second Aerosol Characterization Experiment (ACE-2) stratocumulus case measurements were also analyzed. However, they have not been included here because the particle counter used during ACE-2 has an upper boundary too low $(350 \mu \mathrm{m})$ to cover the complete range of raindrop diameters. Indeed, the drop number in the last bin was often nonzero indicating that the spectra were truncated. However, the results obtained by analyzing the ACE- 2 data set are in agreement with the RICO spectra typical of drizzle (Fig. 5a-f), i.e., with $\sigma_{\mathrm{g}}^{*}$ values on the order of 1.5 and $v^{*}$ values on the order of 5. Moreover, these values are quantitatively consistent with van Zanten et al. (2005) second Dynamics and Chemistry of Marine Stra- tocumulus (DYCOMS-II) stratocumulus $2 \mathrm{~m}$ averaged spectra.

Because samples are mainly in clouds or close to clouds, trade-off values derived in this study may be more representative of the first stages of rain development than of subcloud layer rain spectra. However, because these large drops reach the ground and are not subject to complete evaporation, it may be more important to represent the drop size distribution in the upper levels of the cloud layer in order to accurately represent the raindrop growth and evaporation. If raindrops are size-sorted during their fall and spectra narrower than predicted, it will lead to an overestimation of the fall velocity. However, evaporation of a large raindrop is low because its lifetime in subsaturated air is short. A $2 \mathrm{~mm}$ drop falling in an $80 \%$ relative humidity environment covers a distance of $2 \mathrm{~km}$ in $4 \mathrm{~min}$ and loses only $3 \%$ of its mass. In contrast, a $200 \mu \mathrm{m}$ drop in the same conditions evaporates completely after $11 \mathrm{~min}$ and after a distance of about $700 \mathrm{~m}$. Thus, the predicted amount of rain that evaporates and the amount of precipitation that reaches the ground would not be considerably biased.

As for cloud droplet spectra (G10), the shape parameter is mostly sensitive to the water content as shown by Figure $5 \mathrm{e}$, f. However the size sorting process also modulates the drop spectral width. For samples with low $q_{\mathrm{r}}$, spectra are predominantly narrow (low $1 / \nu$ ) whatever the value of $N_{\mathrm{r}}$. For samples with large $q_{\mathrm{r}}$, the spectra are predominantly broad for large $N_{\mathrm{r}}$ and narrow for small $N_{\mathrm{r}}$ due to size sorting. Thus we parameterize the shape parameter as a function of a power law of $q_{\mathrm{r}}$ and $N_{\mathrm{r}}$. Figure 6a, b show scatterplots of $v$ and $\sigma_{g}$ as a function of $\left(N_{\mathrm{r}} q_{\mathrm{r}}^{0.25}\right)$ and $N_{\mathrm{r}} q_{\mathrm{r}}^{0.1}$, respectively, for the four moments and the values that minimize both absolute and relative errors in each bin. The percentile intervals indicate that the data dispersion increases as $\left(N_{\mathrm{r}} q_{\mathrm{r}}\right)$ increases, especially for moments $M_{1}$ and $M_{2}$. This is consistent with Fig. 5a-d that reveal that the spread of $v$ is larger for large values of $N_{\mathrm{r}}$ while it remains constant over the $q_{\mathrm{r}}$ range.

For each law, the resulting 80 optimum parameters are fitted, which leads to the following expressions:

$v^{p}=18 /\left(N_{\mathrm{r}} q_{\mathrm{r}}\right)^{0.25}$,

$\sigma_{\mathrm{g}}^{p}=1 .+0.30 \cdot\left(N_{\mathrm{r}} q_{\mathrm{r}}\right)^{0.1}$,

where $q_{\mathrm{r}}$ is expressed in $\mathrm{g} \mathrm{m}^{-3}$ and $N_{\mathrm{r}}$ in $\mathrm{m}^{-3}$.

In order to compare the accuracy of each analytical distribution in representing rain spectra, relative and absolute errors between measured and theoretical moments are calculated. Table 2 summarizes the offsets and standard deviations of the absolute and relative errors over the whole range of moment values calculated for the gamma and the lognormal distribution, with trade-off and parameterized values. Both laws give similar results. The parameterized expressions improve the results in terms of both bias and standard deviation. 

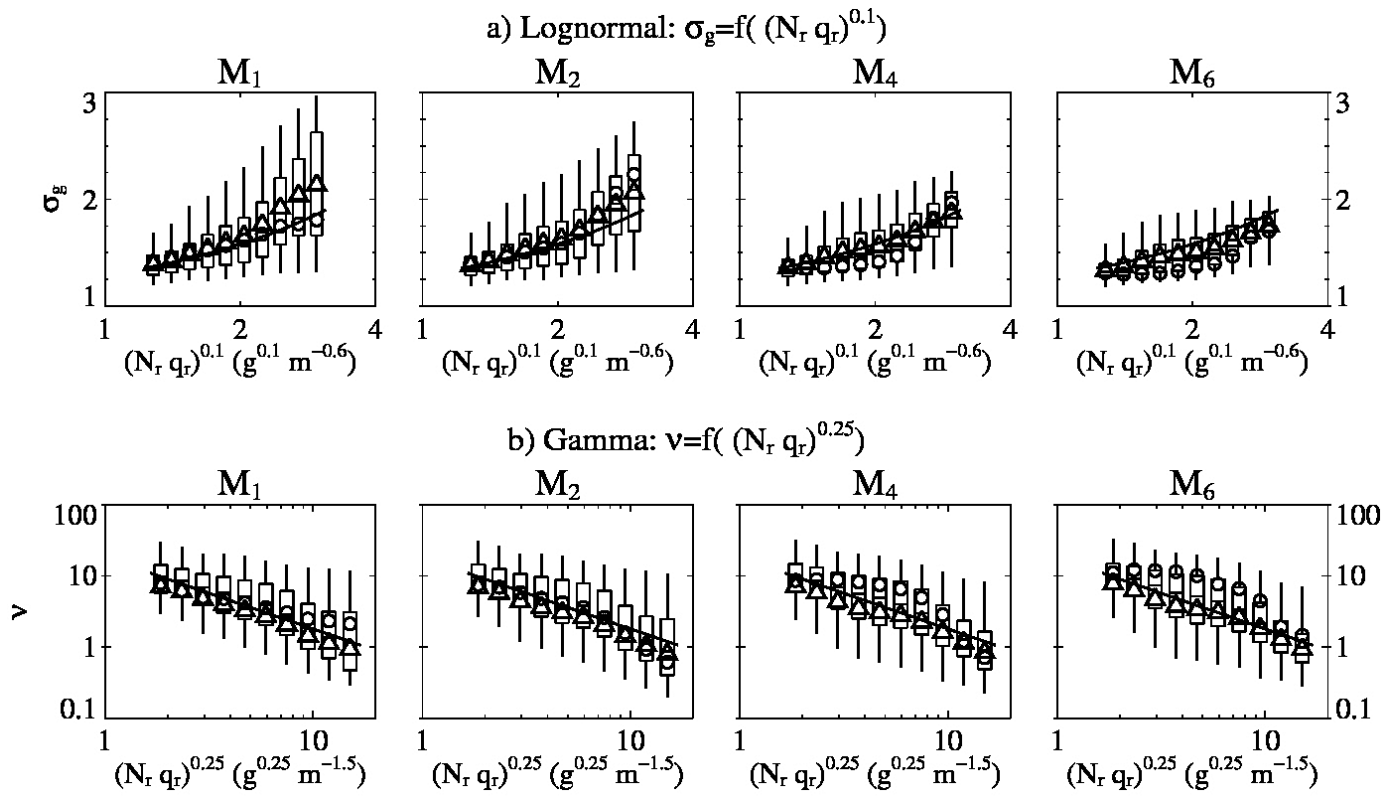

Figure 6. Same as Fig. 4 but plotted as a function of a power law of $q_{\mathrm{r}} N_{\mathrm{r}}$. The thick lines represent the proposed parameterizations for the variable shape parameter.

\section{Conclusions}

In situ measurements of rain collected during the RICO experiment were analyzed in order to validate the commonly used analytical representation of raindrop size distribution and quantify their broadness for shallow cumulus clouds. Data from the PMS OAP-260-X and the PMS 2DP were combined to retrieve the complete raindrop size distribution. Thirteen flights with significant rain events have been selected. The aircraft sampling strategy provides a comprehensive set of raindrop spectra typical of trades shallow cumulus clouds by flying at different levels in the lower troposphere. First, the vertical profiles of the microphysical rain variables were examined. It was shown that the rain number concentration and the mean volume diameter decrease and increase, respectively, with decreasing altitude, whereas the rainwater content remains more or less constant. Both box plots with percentiles of the distribution of the observations and flight average values follow the same pronounced vertical trend, reflecting the consistency of the observations.

Next, the broadness of the size distribution was studied by analyzing the relationship between a considered moment of the size distribution and the two main rain variables used in microphysical schemes: the rain mixing ratio and the rain number concentration. For each moment representative of a physical process $M_{1}, M_{2}, M_{4}$ and $M_{6}$, the shape parameter is calculated numerically by minimizing the distance between the measured moment and the derived analytical moment. For a given spectra, there is generally not a single value of the shape parameter that accurately represents each moment simultaneously. As a general trend, we observe that spectra become narrower as the value of the considered moment increases. Nevertheless, a constant trade-off value is proposed for both the gamma law and the lognormal law. On the ensemble, spectra are found to be broad on the scale of a LES simulation $(\sim 100 \mathrm{~m})$, with trade-off values $v^{*}$ on the order of 3.2 and $\sigma_{\mathrm{g}}^{*}$ on the order of 1.63. On a coarser scale, distributions tend to be broader, with values of the shape parameter close to the MP value, which reflects the heterogeneity of the raindrop field. Given the differences in the altitude of the samples, as well as instrumental issues, these results are consistent with studies in the literature focusing on deep convective events. Tests regarding the sensitivity to extreme values of the drop sizes suggest that the contribution of the smallest drops to the broadness of the distribution is important. The lognormal and the gamma laws give similar results. However, the gamma law allows analytical integration - for instance, the integration of the sedimentation flux using the parameterization of Roger et al. (1993) of the terminal velocity.

As a second step, the dependency of the shape parameter on the variables predicted by a LES microphysical scheme was explored. Measurements show a clear negative trend as function of the rainwater content, but no obvious trend as function of the drop concentration or of the mean volume diameter. These results are consistent with the microphysical processes involved. Indeed, at the early stage of the rain formation, samples are characterized by high-concentration values, low mean volume diameter values and narrow spectra. As drops grow by collision and coalescence, rain becomes more intense and the size spectra broaden. Finally, the rain spectra tend to be narrower near the surface due to 
Table 2. Values of the geometric mean $\mu_{\log }$ and the geometric standard deviation $\sigma_{\log }$ of the log errors, and the arithmetic mean $\mu_{\mathrm{abs}}$ and the arithmetic standard deviation $\sigma_{\mathrm{abs}}$ of the absolute errors calculated for $M_{1}, M_{2}, M_{4}$ and $M_{6}$, for the lognormal and the gamma parametric functions, when using the constant trade-off tuning parameters values, $\sigma_{\mathrm{g}}^{*}$ and $v^{*}$, and the parameterized value as a function of $N_{\mathrm{r}} q_{\mathrm{r}}, \sigma_{\mathrm{g}}^{p}$ and $v^{p}$.

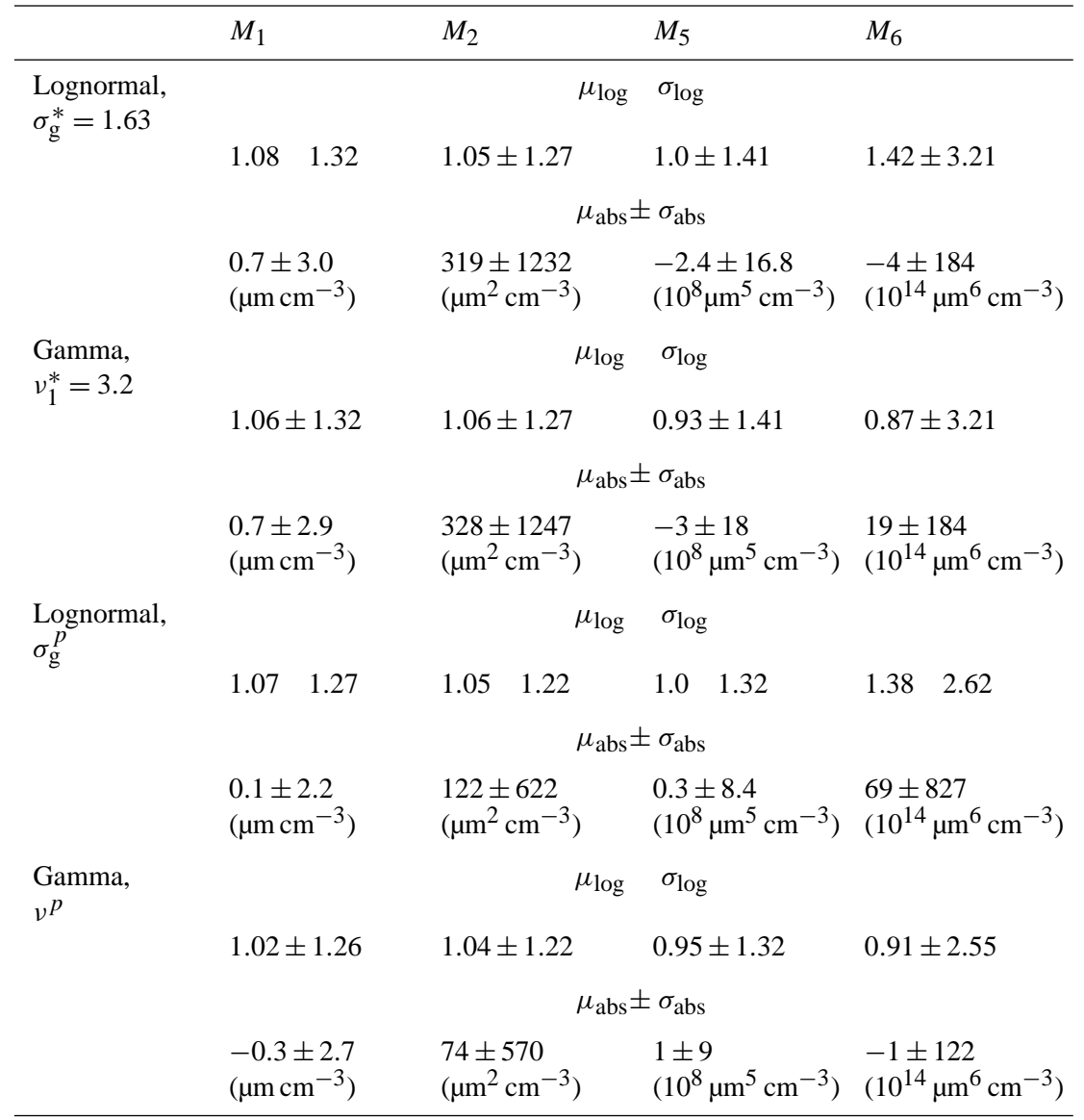

size sorting. In order to take into account this behavior, a parameterization as a function of a power law of $\left(q_{\mathrm{r}} N_{\mathrm{r}}\right)$ that improves the representation of the rain spectra was developed for the LES scale. However, LES simulations of precipitating shallow cumulus clouds showed that a change in $v$ from 1 to 11 impacts the mean liquid water path by about $20 \%$ after 2 to $6 \mathrm{~h}$ of simulations (not shown). These tests also suggested that the use of the trade-off value should be sufficient to represent the magnitude of the precipitation rate in shallow cumulus clouds. Questions remain for deep convection. Indeed a variable shape parameter may impact the results in heavily precipitating clouds significantly (Shipway and Hill, 2012). Moreover, the measurements of raindrop spectra are somehow limited by statistics issues due to the low number of raindrops and by instrumental biases. These measurements are important for reconstructing rain history in the lower troposphere and subsequently for constraining rain formation the main source of uncertainty in precipitation calculation - on the scale of the cloud system. The results presented here highlight the need to improve particle measurements over the whole spectrum range as well as to provide such data at all stages of rain development.

Acknowledgements. We are grateful to two anonymous reviewers for their comments and suggestions that helped to improve the manuscript. We also thank Jean-Louis Brenguier, Odile Thouron, Axel Seifert and Bjorn Stevens for helpful discussions on this topic.

Edited by: J. Quaas 


\section{References}

Baker, B., Mo, Q., Lawson, R. P., O'Connor, D., and Korolev, A.: Drop size distributions and the lack of small drops in RICO rain shafts, J. Appl. Meteor. Climatol., 48, 616-623, 2009.

Best, A. C.: The Size Distribution of Raindrops, Quat. J. Roy. Meteor. Soc., 76, 16-36, 1950.

Brandes, E. A., Zhang, G., and Vivekanandan, J.: An evaluation of a drop distribution-based polarimetric radar rainfall estimator, J. Appl. Meteor., 42, 652-660, 2003.

Burnet, F. and Brenguier, J. L.: The onset of precipitation in warm convective clouds: A case study from SCMS, Quat. J. Roy. Meteor. Soc., 136, 374-381, doi:10.1002/qj.552, 2010.

Cerro, C., Codina, B., Bech, J., and Lorente, J.: Modeling Raindrop Size Distribution and $\mathrm{Z}(\mathrm{R})$ Relations in the Western Mediterranean Area, J. Appl. Meteor., 36, 1470-1479, 1997.

Feingold, G., and Levin, Z.: The lognormal fit to raindrop spectra from frontal convective clouds in Israel. J. Climate Appl. Meteor. 25:1346-1363, 1986.

Chandrasekar, V., Cooper, W. A., and Bringi, V. N.: Axis ratios and oscillations of raindrops, J. Atmos. Sci., 45, 1323-1333, 1988.

Chang, W. Y., Chen Wang, T. C., and Lin, P. L.: Characteristics of the Raindrop Size Distribution and Drop Shape Relation in Typhoon Systems in the Western Pacific from the 2D Video Disdrometer and NCU C-Band Polarimetric Radar, J. Atmos. Ocean. Technol., 26, 1973-1993, 2009.

Geoffroy, O., Brenguier, J.-L., and Sandu, I.: Relationship between drizzle rate, liquid water path and droplet concentration at the scale of a stratocumulus cloud system, Atmos. Chem. Phys., 8, 4641-4654, doi:10.5194/acp-8-4641-2008, 2008.

Geoffroy, O., Brenguier, J.-L., and Burnet, F.: Parametric representation of the cloud droplet spectra for LES warm bulk microphysical schemes, Atmos. Chem. Phys., 10, 4835-4848, doi:10.5194/acp-10-4835-2010, 2010.

Heus, T., van Heerwaarden, C. C., Jonker, H. J. J., Pier Siebesma, A., Axelsen, S., van den Dries, K., Geoffroy, O., Moene, A. F., Pino, D., de Roode, S. R., and Vilà-Guerau de Arellano, J.: Formulation of the Dutch Atmospheric Large-Eddy Simulation (DALES) and overview of its applications, Geosci. Model Dev., 3, 415-444, 2010.

Heymsfield, A. J. and Parrish, J. L.: A Computational Technique for Increasing the Effective Sampling Volume of the PMS TwoDimensional Particle Size Spectrometer, J. Appl. Meteor., 17, 1566-1572, 1978.

Heymsfield, A. J. and Baumgardner, D.: Summary of a workshop on processing 2-D probe data, Bull. Amer. Meteor. Soc., 66, 437$440,1985$.

Hu, Z. and Srivastava, R. C.: Evolution of raindrop size distribution by coalescence, breakup, and evaporation: Theory and observations, J. Atmos. Sci., 52, 1761-1783, 1995.

Jiang, H., Feingold, G., and Sorooshian, A.: Effect of Aerosol on the Susceptibility and Efficiency of Precipitation in Warm Trade Cumulus Clouds, J. Atmos. Sci., 67, 3525-3540, 2010.

Joss, J. and Waldvogel, A.: Raindrop size distribution and sampling size errors, J. Atmos. Sci., 26, 566-569, 1969.

Kessler, E.: On the Distribution and Continuity of Water Substance in Atmospheric Circulation, Meteor. Monogr., No. 32, Amer. Meteor. Soc., 32, 1-84, 1969.

Marshall, J. S. and Palmer, W.: The distribution of raindrops with size, J. Meteor., 5, 165-166, 1948.
Milbrandt, J. and Yau, M.: A multimoment bulk microphysics parameterization. Part I: Analysis of the role of the spectral shape parameter, J. Atmos. Sci., 62, 3051-3064, 2005.

Nuijens, L., Stevens, B., and Siebesma, A.P.: The environment of precipitating shallow cumulus convection, J. Atmos. Sci., 66, 1962-1979, 2009.

Nzeukou, A., Sauvageot, H., Ochou, A. D., Kebe, C. M. F.: Raindrop Size Distribution and Radar Parameters at Cape Verde, J. Appl. Meteor., 43, 90-105, 2004.

Pruppacher, H. R., and Beard, K. V.: A wind tunnel investigation of the internal circulation and shape of water drops falling at terminal velocity in air, Quart. J. Roy. Meteor. Soc., 96, 247-256, 1970.

Pruppacher, H. R., and Pitter, R. L.: A semi-empirical determination of the shape of cloud and raindrops, J. Atmos. Sci., 28, 86-94, 1971.

Seifert, A.: On the Parameterization of Evaporation of Raindrops as Simulated by a One-Dimensional Rainshaft Model, J. Atmos. Sci., 65, 3608-3619, 2008.

Tokay, A. and Short, D. A.: Evidence from tropical raindrop spectra of the origin of rain from stratiform and convective clouds, J. Appl. Meteor. 35, 355-371, 1996.

Uijlenhoet, R., Steiner, M., and Smith, J. A.: Variability of raindrop size distributions in a squall line and implications for radar rainfall estimation, J. Hydrometeor., 4, 43-61, 2003.

Ulbrich, C. W.: Natural variations in the analytical form of the raindrop size distribution, J. Climate Appl. Meteor., 22, 1764-1775, 1983.

Ulbrich, C. W. and Atlas, D.: Rainfall microphysics and radar properties: Analysis methods for drop size spectra, J. Appl. Meteor., 37, 912-923, 1998.

Rauber, R. M., Ochs III, H. T., Di Girolamo, L., Göke, S., Snodgrass, E., Stevens, B., Knight, C., Jensen, J. B., Lenschow, D. H., Rilling, R. A., Rogers, D. C., Stith, J. L., Albrecht, B. A., Zuidema, P., Blyth, A. M., Fairall, C. W., Brewer, W. A., Tucker, S., Lasher-Trapp, S. G., Mayol-Bracero, O. L., Vali, G., Geerts, B., Anderson, J. R., Baker, B. A., Lawson, R. P., Bandy, A. R., Thornton, D. C., Burnet, E., Brenguier, J-L., Gomes, L., Brown, P. R. A., Chuang, P., Cotton, W. R., Gerber, H., Heikes, B. G., Hudson, J. G., Kollias, P., Krueger, S. K., Nuijens, L., O’Sullivan, D. W., Siebesma, A. P., and Twohy, C. H.: Rain in (shallow) cumulus over the ocean - the RICO campaign, B. Am. Meteorol. Soc., 88, 1912-1928, 2007.

Rogers, R. R., Baumgardner, D., Ethier, S. A., Carter, D. A., and Ecklund, W. L.: Comparison of raindrop size distributions measured by radar wind profiler and by airplane, J. Appl. Meteor., 32, 694-699, 1993.

Seifert, A.: On the parameterization of evaporation of raindrops as simulated by a one-dimensional rainshaft model, J. Atmos. Sci., 65, 3608-3619, 2008.

Seifert, A., and Beheng, K. D.: A double-moment parameterization for simulating autoconversion, accretion, and self-collection, Atmos. Res., 59-60, 265-281, 2001.

Shipway, B. J. and Hill, A. A. : Diagnosis of systematic differences between multiple parametrizations of warm rain microphysics using a kinematic framework, Q. J. R. Meteorol. Soc., 138, 2196 2211, doi:10.1002/qj.1913, 2012.

Smith, P. L., Myers, C. G., and Orville, H. D.: Radar Reflectivity Factor Calculations in Numerical Cloud Models Using Bulk Pa- 
rameterization of Precipitation, J. Appl. Meteor., 14, 1156-1165, 1975.

Snodgrass, E. R., Girolamo, L. D., and Rauber, R. M.: Precipitation characteristics of trade wind clouds during RICO derived from radar, satellite, and aircraft measurements, J. Appl. Meteor., 48, 464-483, 2009.

Stevens, B. and Seifert, A.: Understanding macrophysical outcomes of microphysical choices in simulations of shallow cumulus convection, J. Meteor. Soc. Japan, 86, 143-162, 2008.

van Zanten, M. C., Stevens, B.,. Vali, G., and Lenschow, D. H.: Observations of drizzle in nocturnal marine stratocumulus. J. Atmos. Sci., 62, 88-106, 2005.

van Zanten, M., Stevens, B., Nuijens, L., Siebesma, A., Ackerman, A., Burnet, F., Cheng, A., Couvreux, F., Jiang, H., Khairoutdinov, M., Kogan, Y., Lewellen, D., Mechem, D., Nakamura, K., Noda, A., Shipway, B., Slawinska, J., Wang, S., and Wyszogrodzki, A.: Controls on precipitation and cloudiness in simulations of tradewind cumulus as observed during RICO, J. Adv. Model. Earth Syst., 3, doi:10.1029/2011MS000056, 2011.
Waldvogel, A.: The $\mathrm{N}_{0}$ jump of raindrop spectra, J. Atmos. Sci.,31, 1067-1078, 1974.

Wood, R.: Drizzle in Stratiform Boundary Layer Clouds. Part I: Vertical and Horizontal Structure, J. Atmos. Sci., 62, 3011-3033, 2005.

Yuter, S. E., and Houze, R. A.: Measurements of raindrop size distributions over the Pacific warm pool and implications for Z-R relations, J. Appl. Meteor., 36, 847-867, 1997.

Zhang, G., Vivekanandan, J., and Brandes E.: A method for estimating rain rate and drop size distribution from polarimetric radar measurements, IEEE Trans. Geosci. Remote Sens., 39, 830-841, 2001. 